\title{
Body Weight Variability: A Marker for Increased Risk or a Causative Factor for Dementia?
}

\author{
Yong-ho Lee L, 2,* $^{2}$ \\ ${ }^{1}$ Department of Internal Medicine and ${ }^{2}$ Institute of Endocrine Research, Yonsei University College of \\ Medicine, Seoul, Korea
}

\author{
Received August 25, 2021 \\ Accepted September 12, 2021 \\ ${ }^{*}$ Corresponding author \\ Yong-ho Lee \\ https://orcid.org/0000-0002-6219-4942 \\ Department of Internal Medicine, Yonsei \\ University College of Medicine, 50-1 \\ Yonsei-ro, Seodaemun-gu, Seoul 03722, \\ Korea \\ Tel.: +82-2-2228-1943 \\ Fax: +82-2-393-6884 \\ E-mail:yholee@yuhs.ac
}

Dementia is an emerging burden associated with significant morbidity and mortality in aging populations. In recent decades, dementia prevalence has continually increased in Korea ${ }^{1}$ as well as globally and is showing no signs of slowing down burden. ${ }^{2}$ Dementia is often accompanied by immobility and malnutrition, and eventually requires significant support from others; in fact, the medical care costs for individuals with dementia are remarkably greater than those for cancer, heart disease, or other chronic diseases. ${ }^{3}$ In this context, risk factors for the development of dementia are of significant interest as they can be lead to prevention and management to mitigate this growing epidemic.

A number of modifiable and nonmodifiable risk factors for cognitive decline and dementia have been well identified and verified. These include aging, diabetes, smoking, low physical activity, sedentary lifestyle, poor diet, excess alcohol consumption, family history, apolipoprotein E4 phenotype, midlife obesity, midlife high cholesterol, hypertension, depression, and lower educational level. ${ }^{4}$ In terms of body weight, underweight and obesity at midlife are associated with an elevated risk of dementia, ${ }^{5,6}$ whereas reversed causation exists between dementia risk and late life obesity. ${ }^{6}$ Recently, obese individuals with normal metabolic profiles, called metabolically healthy obese, in late life demonstrated lower risk of overall dementia and Alzheimer disease, while obese individuals with abnormal metabolic profiles, called metabolically unhealthy obese, had increased risk of Alzheimer's disease, indicating a complex relationship between obesity status and dementia risks. ${ }^{7}$ In addition, weight loss usually precedes clinical diagnosis of dementia by approximately 10 years. ${ }^{8}$

However, most studies are based on body mass index (BMI) measured at baseline, without any data over follow-up despite its variability. As an individual's body weight often varies over time, this may affect the risk of developing dementia or cognition-related disorders. Bae and Park ${ }^{9}$ investigated the association between variability in BMI with the risk of developing cognitive impairment using an aging cohort Korean Longitudinal Study of Aging over a follow-up of approximately 6 years. In this well-established database of 3,477 individuals who had no evidence of cognitive impairment at baseline, biennial BMI measurements were recorded and cognitive performance was assessed by the Korean version of the MiniMental State Examination (K-MMSE). Two separate methods were applied to evaluate individual variability in body weight: (1) objective index: average successive variability (ASV) defined as the average of absolute difference in BMI between three successive measurements and (2) subjective index: self-reported frequency of 
weight changes over $5 \mathrm{~kg}$ during the past year. For example, an ASV of 1 indicates either an increase or a decrease of $1 \mathrm{~kg} / \mathrm{m}^{2}$ in BMI. Individuals with the highest variation in BMI or a high frequency (more than three times) of self-reported weight changes had a significantly higher likelihood of developing cognitive impairment with odds ratio of 1.52 and 3.42, respectively. Furthermore, regardless of the overall weight change trends, self-reported frequency of weight changes showed consistent association with progression of cognitive impairment. Although there was no significant association between ASV and severe cognitive impairment (K-MMSE $\leq 17$ ), this is not surprising because the numbers of individuals who developed severe cognitive impairment were relatively small due to the short duration of follow-up time and relatively young participants ( $27.8 \%$ of the study population were aged over 65 years).

Recent accumulating evidence, including the present proposal, shows that body weight variability may have a role in predicting incident dementia ${ }^{10,11}$ or cognitive impairment, ${ }^{9}$ independent from baseline status of obesity. Although the underlying mechanism between body weight variability and cognitive decline has not been investigated in humans yet, a mouse study showed that weight fluctuations aggravated hepatic steatosis, insulin resistance, and adipose tissue dysfunction, which are important contributors for dementia. ${ }^{12}$ Regarding body composition, regained body weight predominately consists of fat, whereas weight loss is usually caused by losing both fat and fat-free tissue, including muscle. ${ }^{13}$ A major limitation of BMI is that it cannot differentiate between fat and lean body mass, and involuntary loss of skeletal muscle followed by compensatory consumption of high calorie food may result in sarcopenia or sarcopenic obesity, which are recently reported to have significant relationships with impaired cognitive function in a longitudinal dataset of elderly individuals. ${ }^{14}$

Comparing with two previous retrospective studies, ${ }^{10,11}$ which used relatively inaccurate information of International Classification of Diseases, tenth revision (ICD-10) codes with or without prescription data of antidementia drug for defining dementia, the present study analyzed objective methods for evaluating cognitive function (K-MMSE) from well-established prospective cohort data. ${ }^{9}$ Moreover, using two independent definitions of body weight variability has improved the consistency of the study findings.
However, there are several limitations that should be complemented by future research. As Bae and Park ${ }^{9}$ did not show differences in K-MMSE at baseline by ASV and baseline K-MMSE scores were not adjusted in the logistic models, this finding should be interpreted with caution. Considering that individuals with the greatest quintile of ASV were likely to have many of the dementia risk factors in Table 1 of Ref. 9, they may have lower K-MMSE scores at baseline, which would lead to more rapid progression of cognitive decline. Analyzing the association of body weight variability with cognitive impairment after stratification of the study population by obesity status at baseline would provide more information regarding complex relationship between obesity and weight fluctuation. The authors ${ }^{9}$ described the mean values of BMI changes in the lowest (Q1) and greatest (Q5) quintile of ASV, which were 0.2 and 2.7, respectively, during 4 years. Exploring the optimal cut off points of ASV will provide more practical information to clinicians to assess dementia risk based on body weight variability.

This study has contributed to our understanding of the risk of dementia progression in Asian populations. Although further investigations are necessary for determining whether body weight variability itself can cause incident dementia or act as a predictive marker for dementia, it is important for health care providers to consider body weight variability as a potential indicator for identifying high risk patients with dementia. In an era of big data and artificial intelligence, integrating patients' longitudinal data of body weight and interpreting their variability to assess individuals' risks for diseases, including dementia, is a plausible approach.

\section{CONFLICTS OF INTEREST}

Yong-ho Lee is an editorial board member of the journal, but he was not involved in the peer reviewer selection, evaluation, or decision process of this article. Otherwise, no other potential conflicts of interest relevant to this article were reported.

\section{REFERENCES}

1. Jang JW, Park JH, Kim S, Lee SH, Lee SH, Kim YJ. Prevalence and incidence of dementia in South Korea: a nationwide analysis of the National Health Insurance Service Senior Cohort. 
J Clin Neurol 2021;17:249-56.

2. Ferri CP, Prince M, Brayne C, Brodaty H, Fratiglioni L, Ganguli M, et al. Global prevalence of dementia: a Delphi consensus study. Lancet 2005;366:2112-7.

3. Kelley AS, McGarry K, Gorges R, Skinner JS. The burden of health care costs for patients with dementia in the last 5 years of life. Ann Intern Med 2015;163:729-36.

4. Peters R, Booth A, Rockwood K, Peters J, D’Este C, Anstey KJ. Combining modifiable risk factors and risk of dementia: a systematic review and meta-analysis. BMJ Open 2019;9:e022846.

5. Whitmer RA, Gunderson EP, Barrett-Connor E, Quesenberry CP Jr, Yaffe K. Obesity in middle age and future risk of dementia: a 27 year longitudinal population based study. BMJ 2005;330:1360.

6. Fitzpatrick AL, Kuller LH, Lopez OL, Diehr P, O’Meara ES, Longstreth WT Jr, et al. Midlife and late-life obesity and the risk of dementia: cardiovascular health study. Arch Neurol 2009;66:336-42.

7. Lee JY, Han K, Han E, Kim G, Cho H, Kim KJ, et al. Risk of incident dementia according to metabolic health and obesity status in late life: a population-based cohort study. J Clin Endocrinol Metab 2019;104:2942-52.

8. Knopman DS, Edland SD, Cha RH, Petersen RC, Rocca WA. Incident dementia in women is preceded by weight loss by at least a decade. Neurology 2007;69:739-46.

9. Bae EM, Park SM. Association between variations in body mass index and cognitive function in older Korean adults. J Obes Metab Syndr 2021;30:271-8.

10. Roh E, Hwang SY, Kim JA, Lee YB, Hong SH, Kim NH, et al. Body weight variability increases dementia risk among older adults: a nationwide population-based cohort study. Front Endocrinol (Lausanne) 2020;11:291.

11. Kang SY, Kim YJ, Jang W, Son KY, Park HS, Kim YS. Body mass index trajectories and the risk for Alzheimer's disease among older adults. Sci Rep 2021;11:3087.

12. Zamarron BF, Porsche CE, Luan D, Lucas HR, Mergian TA, Martinez-Santibanez G, et al. Weight regain in formerly obese mice hastens development of hepatic steatosis due to impaired adipose tissue function. Obesity (Silver Spring) 2020;28: 1086-97.

13. van der Kooy K, Leenen R, Seidell JC, Deurenberg P, Hautvast JG. Effect of a weight cycle on visceral fat accumulation. Am J Clin Nutr 1993;58:853-7.

14. Batsis JA, Haudenschild C, Roth RM, Gooding TL, Roderka MN, Masterson T, et al. Incident impaired cognitive function in sarcopenic obesity: data from the national health and aging trends survey. J Am Med Dir Assoc 2021;22:865-72.e5. 